\title{
RESSECÇÃO HEPÁTICA COM USO DE RADIOFREQÜÊNCIA BIPOLAR
}

\author{
HEPATIC RESECTION WITH THE USE OF BIPOLAR RADIOFREQUENCY
}

\author{
Fábio Luiz Waechter - TCBC-RS ${ }^{\mathbf{~}}$; José Artur Sampaio'; \\ Paulo Renê Soares Berger²; Eliézer Knob de Souza²
}

\begin{abstract}
RESUMO: Os autores apresentam uma nova técnica de hepatectomia através de agulhas paralelas de radiofrequiência bipolar. Abordando o impacto trans-operatório, assim como a evolução pós-operatória dos pacientes (Rev. Col. Bras. Cir. 2007; 34(5): 340-342).

Descritores: Hepatectomia; Fígado; Ablação por Cateter.
\end{abstract}

\section{INTRODUÇÃO}

Com o intuito de minimizar as complicações da cirurgia hepática(sangramento, a fístula biliar e a manutenção da função hepática pós-ressecção), os autores desenvolveram uma nova técnica para ressecar parênquima hepático através de pequenas laparotomias, sem necessidade de clampeamento vascular, com mínima(ou ausência) dissecção hepática, ou sangramento. Para tanto, agulhas de radiofrequiência (RF) refrigeradas, tendo como fonte de energia um eletrocautério bipolar, foram criadas, tendo sido utilizadas de rotina pelos autores no presente Serviço de Cirurgia.

\section{Características construtivas e funcionais das agu-}

\section{lhas de RF}

O sistema de agulhas montado pelos autores apresenta dois eletrodos paralelos ( 18 gauge cada) de 25 centímetros $(\mathrm{cm})$ de comprimento, separados entre si por uma distância de $1 \mathrm{~cm}$, onde apenas os $4 \mathrm{~cm}$ distais dissipam energia gerada por um eletrobisturi bipolar (Figura 1). Estas agulhas são refrigeradas através de um sistema de resfriamento interno por fluxo contínuo com soro gelado estéril a $0^{\circ}$ Celsius(C).

O eletrobisturi funciona como fonte da energia elétrica que gera a corrente elétrica necessária para a desnaturação do tecido-alvo. No caso do dispositivo usado, a freqüência nominal é de $500 \mathrm{kHz}$ modulada por $30 \mathrm{kHz}$. A potência disponível pelo aparelho fica entre 4 e 80 watts. Este aparelho é dotado de controle de potência aplicada e de dispositivo de monitoramento da resistência do tecido entre os eletrodos, desligando o aparelho quando a resistência subir acima de um limite de segurança que evita a cauterização e necrose total do tecido.

O objetivo é gerar uma área de tecido desnaturado (Figura 2) propícia a criar uma linha de corte por meio de um

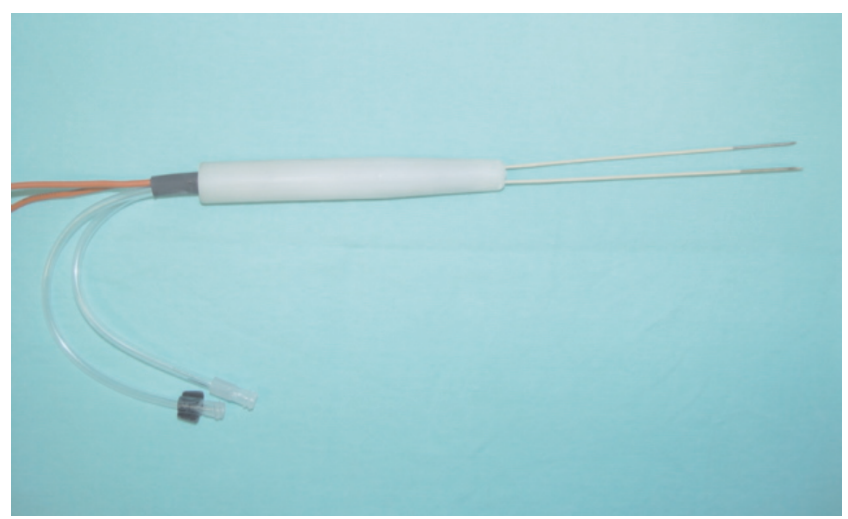

Figura 1 - Manopla com duas agulhas refrigeradas de RF.

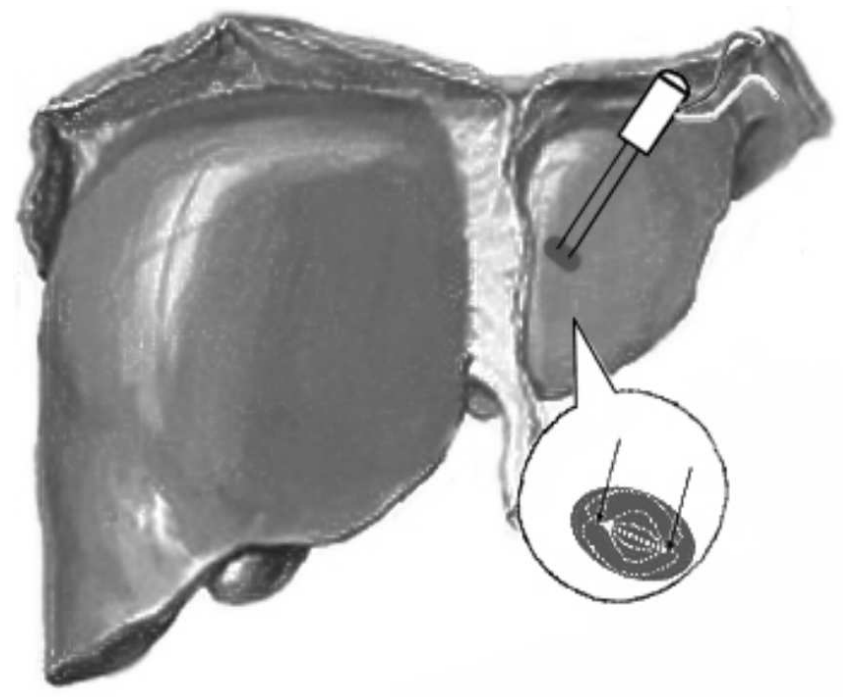

Figura 2 - Utilização das agulhas de RF criando área de necrose hepática para posterior secção exangue com bisturi de lâmina convencional.

1. Cirurgião do Aparelho Digestivo do Hospital Moinhos de Vento - Porto Alegre - RS

2. Engenheiro Biomédico do Hospital Moinhos de Vento - Porto Alegre - RS.

Recebido em 05/06/2007

Aceito para publicação em 03/08/2007

Conflito de interesses: nenhum

Fonte de financiamento: nenhuma

Trabalho realizado Serviço de Cirurgia e Engenharia Biomédica - - Brasil 
bisturi de lâmina convencional sem sangramento. A área de necrose compreende $1 \mathrm{~cm}$ de largura por $1 \mathrm{~cm}$ de extensão por 4 $\mathrm{cm}$ de profundidade, deixando apenas $0,5 \mathrm{~cm}$ de tecido desnaturado junto ao parênquima remanescente, já que a secção com lâmina convencional é executada junto a borda das agulhas que estão em contato com a peça a ser removida. Esta área ocorre exatamente entre os eletrodos ativos, num volume que se estende até cerca de $80 \%$ da parte ativa exposta.

A tensão elétrica alternada gerada pelo eletrobisturi é aplicada pelos eletrodos e gera um gradiente de densidade de corrente elétrica na região entre os eletrodos. Como o tecido da região é composto por células com determinada concentração de íons em solução aquosa, peculiar ao volume intracelular, a circulação da corrente elétrica tem baixa resistência no início do processo. Entretanto, a manutenção da circulação desta corrente alternada faz com que a resistência subitamente baixe, intensificando o calor gerado pelo efeito Joule. Este aquecimento faz com que o tecido comece a desnaturar, com conseqüente perda de líquido, gerando um aumento gradativo da resistência do tecido entre os eletrodos ${ }^{1,2}$. Isto ocorre até alcançar níveis em que há progressiva diminuição do efeito Joule. No momento em que a resistência alcança níveis de segurança pré determinados, o eletrobisturi desliga a fonte de tensão. A temperatura gerada por este efeito chega a cerca de $80^{\circ} \mathrm{C}$ no tecido ${ }^{2,3}$. Os eletrodos são resfriados para que o tecido em contato com o mesmo não fique necrosado e aderido(os quais aumentam muito a resistência elétrica do tecido), garantindo assim um fluxo contínuo de corrente elétrica entre os mesmos e uma desnaturação mais gradual e uniforme em todo o volume compreendido entre os eletrodos ativos ${ }^{4}$.

\section{Descrição da Técnica Empregada}

Os pacientes são submetidos à anestesia geral, não sendo utilizados cateteres venosos centrais durante ou após o procedimento. As incisões mínimas realizadas são subcostal direita, mediana supra-umbilical, e/ou combinadas.

Com exceção dos pacientes submetidos a hepatectomias direita e esquerda que são dissecados os ligamentos do lado a ser ressecado, mínima, ou nenhuma, mobilização dos ligamentos é realizada. A demarcação da linha de ressecção com eletro-cautério monopolar definia a área a ser removida, permitindo que se obtenha uma distância mínima de $1 \mathrm{~cm}$ do tumor.

Sempre com controle ecográfico, a introdução das agulhas é iniciada pela borda livre do fígado em direção ao interior do parênquima. Uma vez posicionada as agulhas, o processo de coagulação é iniciado, sendo interrompido quando a impedância tecidual elevada é aferida. Neste momento, total coagulação tecidual é obtida entre as agulhas, assim como por uma distância de $0,5 \mathrm{~cm}$ ao redor e no eixo formado entre elas. O parênquima hepático é então seccionado com bisturi de lâmina convencional exatamente no meio da linha de necrose observada, seguindo, tanto em extensão, como em profundidade, até a completa ressecção segmentar, sem necessidade de utilização de qualquer sutura ou cola biológica. Todos os vasos, incluindo vasos maiores (ramos portais e arteriais, veias hepáticas), são seccionados com bisturi convencional após o "cozimento" tecidual com RF. A drenagem da cavidade, ao término da cirurgia, é realizada de maneira seletiva.

\section{DISCUSSÃO}

A morbidade da hepatectomia, referida preferencialmente pelas complicações supra citadas, permanece como o tendão de Aquiles dos cirurgiões de fígado. Com o objetivo de evitá-las, diferentes técnicas tem sido desenvolvidas através de grandes dissecções dos ligamentos hepáticos, cava, e pedículo portal associadas ao uso de diversos tipos de controles ou clampeamentos vasculares.

Entre as tecnologias de vanguarda, é destaque o uso da RF, bem conhecida no armamentário terapêutico ablativo de lesões de fígado ${ }^{5}$. Ampliando o uso da RF, séries recentes 6-8 têm apresentado hepatectomias realizadas com auxílio da RF monopolar, onde a perda sanguínea e a ocorrência de fístulas biliares foi quase nula. Neste sistema monopolar, a necessidade de aterramento com placa é imperativ. Os riscos de queimaduras frente a grande descarga de energia são existentes.

O procedimento aqui descrito é embasado nos estudos físicos e fisiológicos obtidos com o uso da RF associado ao resfriamento da agulha ${ }^{1-4}$. Como diferença fundamental destaca-se o uso de uma manopla com duas agulhas separadas, transmitindo energia entre elas de maneira bipolar, proporcionado uma área linearmente controlada de necrose, sem o risco de dissipação de corrente para tecido à distância, evitando assim lesões de calor. Além disto, verifica-se a necessidade de potências mais baixas (60W versus $100 \mathrm{~W})$, tornando o procedimento mais seguro e tão eficaz quanto a RF utilizada de maneira monopolar.

Este tecido hepático desnaturado forma uma placa de consistência borrachosa, não sendo possível distinguir qualquer estrutura vascular ou biliar. Esta placa formada assegura um selo que não apenas oclui os vasos e canais biliares da linha de secção, mas também sela os pequenos canais linfáticos desta mesma face cruenta. O resultado é a ausência de fístulas biliares associado ao baixíssimo índice de sangramento transoperatório e drenagem obtida nos drenos abdominais no pós-operatório.

Além de tornar a cirurgia muito menos estressante para o cirurgião, a possibilidade do uso de dois conjuntos de agulhas bipolares, contemplando à dois cirurgiões a feitura da ressecção, também permite que ocorra uma drástica redução do tempo cirúrgico, colaborando para uma menor agressividade operatória.

Com relação ao pós-operatório, normalmente observa-se um menor tempo de internação, menos queixas dolorosas e um retorno mais precoce às atividades cotidianas. Isto se explica já que mesmo durante o trans-operatório a cirurgia se torna menos invasiva, sem necessidade de acesso venoso central e com incisões consideravelmente menores, fazendo da hepatectomia um procedimento menos agressivo do ponto de vista de trauma cirúrgico.

Benefício também importante é obtido pela ausência de utilização de materiais de alto custo no trans (sangue e hemoderivados, fios, colas, malhas biológicas, ...) e pós-ope- 
ratório (Centro de Tratamento Intensivo, cateteres para acesso venoso central, ...) permite que o custo hospitalar também diminua drasticamente.

Se por um lado a técnica aqui descrita, além de diversos benefícios gerais, possibilita uma maior tranquiilidade téc- nica na realização da ressecção, é válido frisar que o conhecimento da anatomia hepática e a experiência em ressecções complexas são peças fundamentais no sucesso deste procedimento, sendo ele reservado a cirurgiões afeitos a cirurgia hepática.

\begin{abstract}
To present a new technique of hepatectomy, using parallel bipolar radiofrequency needles, discussing the operative impact as well as the patient's postoperative outcome.
\end{abstract}

Key Words: Liver resection; Liver; Catheter ablation.

\section{REFERÊNCIAS}

1. Haemmerich D, Wright AW, Mahvi DM, Lee FT Jr, Webster JG. Hepatic bipolar radiofrequency ablation creates coagulation zones close to blood vessels: a finite element study. Med Biol Eng Comput. 2003;41(3):317-23.

2. Lounsberry W, Goldschmidt V, Linke CA, Walder HJ, Chrzan D. The early histologic changes following electrocoagulation. J Urol. 1961;86:321-9.

3. Sapareto SA, Dewey WC. Thermal dose determination in cancer therapy. Int J Radiat Oncol Biol Phys. 1984;10(6):787-800.

4. Haemmerich D, Chachati L, Wright AS, Mahvi DM, Lee FT Jr, Webster JG. Hepatic radiofrequency ablation with internally cooled probes: effect of coolant temperature on lesion size. IEEE Trans Biomed Eng. 2003;50(4):493-500.

5. Livraghi T, Solbiati L, Meloni F, Ierace T, Goldberg SN, Gazelle GS. Percutaneous radiofrequency ablation of liver metastases in potential candidates for resection: the "test-of-time approach". Cancer. 2003;97(12):3027-35.

6. Weber JC, Navarra G, Jiao LR, Nicholls JP, Jensen SL, Habib NA. New technique for liver resection using heat coagulative necrosis. Ann Surg. 2002;236(5):560-3.
7. Navarra G, Spalding D, Zacharoulis D, Nicholls JP, Habib NA. Bloodless hepatectomy technique. HPB. 2002;4(1):95-7.

8. Zacharoulis D, Asopa V, Navarra G, Nicholls JP, Jensen SL, Habib NA. Hepatectomy using intraoperative ultrasound-guided radiofrequency ablation. Int Surg. 2003;88(2):80-2.

Como citar este artigo:

Waechter FL, Sampaio JA, Berger PRS Souza EK. Ressecção hepática com uso de radiofrequiência bipolar. Rev Col Bras Cir. [periódico na Internet] 2007 Set-Out;34(5). Disponível em URL: www.scielo.br/ rcbc

Endereço Para Correspondência:

Fábio Luiz Waechter

Rua Ramiro Barcelos 910/605

90035-001 - Porto Alegre - RS

FAX: $0++5133113310$

Telefone: $0++5133122608$

E-mail: waechter@terra.com.br 